\title{
Human and E.coli excinucleases are affected differently by the sequence context of acetylaminofluorene-guanine adduct
}

David Mu, Elisabeth Bertrand-Burggraf ${ }^{1}$, Juch-Chin Huang, Bobert P.P.Fuchs ${ }^{1}$ and Aziz Sancar* Department of Biochemistry and Biophysics, University of North Carolina, School of Medicine, Chapel Hill, NC 2759-79260, USA and 'UPR 9003 Cancerogenese et Mutagenese, Moleculaire et Structurale, CNRS, Ecole Superieure de Biotechnologie de Strasbourg, Boulevard Sebastien Brandt, 67400 Strasbourg/lllkrich-Graffenstaden, France

Received September 26, 1994; Revised and Accepted October 18, 1994

\begin{abstract}
Synthetic DNA substrates containing an acetylaminofluorene (AAF) adduct at each of the three guanine in the $G_{1} G_{2} C G_{3} C C$ sequence were constructed and tested as substrates for reconstituted E.coli (A)BC excinuclease and human excinuclease in HeLa cell-free extract (CFE). The (A)BC excinulcease repaired the three substrates with relative efficiencies of G1:G2:G3 of 100:18:66 in agreement with an ealier report [Seeberg, E., and Fuchs, R.P.P. (1990) Proc. Natl Acad. Sci. USA 87, 191-194]. The same lesions were repaired by the human excinuclease with the strikingly different efficiencies of G1:G2:G3 as 38:100:68. These results reveal that the human excinuclease is affected by the sequence context of the lesion in a different manner than its prokaryotic counterpart.
\end{abstract}

\section{INTRODUCTION}

Mutagenic potential of a DNA adduct depends on several factors ranging from the types of lesions, sequence context of lesion and relative location of the lesion with regard to the replication fork, to the repairability of the lesion $(1,2)$. Analyses of mutation spectra of AAF in E.coli revealed that NarI recognition sequence
(GGCGCC) was a particularly hot spot, with -2 frameshift mutations predominating (3). A study with uniquely modified DNA revealed that all of the mutations at this site arose from the G3-AAF adduct at the NarI sequence (4). Recently these uniquely modified substrates were tested in an in vitro human replication/mutagenesis system (2) and again it was found that only the G3 adduct was mutagenic. Thus the human and E.coli systems behaved nearly identically in terms of mutagenicity for AAF-NarI sequence adducts. Given the similar mutagenic behavior, we wished to know whether in human as well there was a discordance between the repairability and the mutagenicity of AAF lesions at the NarI sequence. The results of this report suggest that repairability is not an important determinant in the mutagenicity of AAF adducts in either humans or E.coli and that, at least in this particular case, the sequence context of the lesion has entirely different effects on human and $E$.coli excinucleases.

\section{MATERIALS AND METHODS}

\section{Substrates}

The substrates were 138-bp duplexes which were constructed by ligating an AAF-adducted 14-nt oligomer with four other oligomers as described previously (5). In the particular batches

Table 1. Extents of excision by human and E.coli excinucleases on AAF adducts at 3 different locations in the same sequence

\begin{tabular}{|c|c|c|c|c|c|c|c|c|}
\hline substrate & $\begin{array}{l}\text { Percent } \\
\text { Human } \\
\text { Exp1 }\end{array}$ & xcised $^{1}$ & Exp3 & $\left(\right.$ Relative $\left.^{2}\right)$ & $\begin{array}{l}\text { E.coli } \\
\text { Exp1 }\end{array}$ & Exp2 & Exp3 & (Relative $^{2}$ ) \\
\hline G1 & 4.0 & 1.6 & 1.3 & 38 & 14.5 & 38.0 & 25.0 & 100 \\
\hline G2 & 7.5 & 4.5 & 3.9 & 100 & 3.7 & 7.0 & 9.0 & 18 \\
\hline G3 & 4.5 & 2.8 & 2.8 & 68 & 10.0 & 25.0 & 14.0 & 66 \\
\hline
\end{tabular}

${ }^{1}$ The reaction mixtures contained ca. $1 \mathrm{nM}$ substrate (identical concentrations for all three substrates) and saturating amount of enzyme as determined experimentally. The actual percent excisions $(<10 \%)$ for the human enzyme are comparable to those of excision assays with other substrates in our laboratory, indicating the superiority of a defined system as in the case of $E$.coli enzyme.

${ }^{2}$ These numbers were obtained by averaging the three experiments, raising the optimal substrate excision level to $100 \%$, and normalizing the other two substrate excision level to that of the optimal.

*To whom correspondence should be addressed 

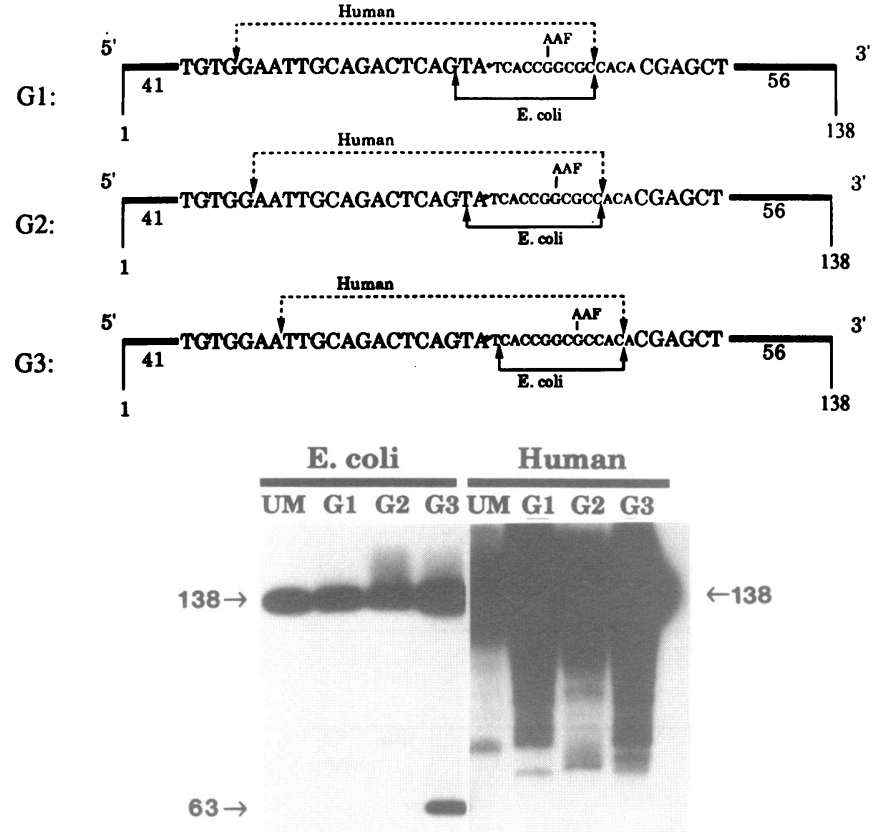

$12 \rightarrow$

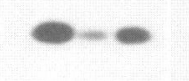

Figure 1. (Top panel) The G-AAF substrates used in this study. The 138-bp duplexes were constructed by ligating the AAF-adducted 14 mer (sequence in lower case) with 4 other oligomers. The ${ }^{32} \mathrm{P}$ label is indicated by an asterisk and the position of the AAF adduct is marked. The brackets indicate the incision sites of the E.coli excinuclease and the extrapolated incision sites of human excinuclease for the longest excision product. (Bottom panel) Excision of G-AAF adducts by $E$.coli and human excinucleases. The lanes $1-4$ contained $1 / 20$ th the amount of DNA loaded into lanes 5-8. The G-AAF is excised in the form of $12 \mathrm{mer}$, consistent with the previous report of Seeberg and Fuchs (1). The band at 63 nt in lane 4 results from excision of the G3 substrate. The numbers $26,27,28$ are the major excision products of human excinuclease although the relative frequency varies depending on the substrate.

of 14-mers used in this study, the G1 and G2 were free of contaminants but G3 substrate was contaminated with $\mathrm{G} 1$ by about $20 \%$ as judged by T4-polymerase assay $(6,7)$. This contamination was attributed to the incomplete separation of G3

oligomer from $\mathrm{G} 1$ oligomer on a $\mathrm{C} 18$ reverse phase column by high performance liquid chromatography.

\section{Enzyme systems}

HeLa whole cell-free extract prepared by the method of Manley et al. (8) was used as a crude source of human excinuclease. The E.coli (A)BC excinuclease was reconstituted from purified UvrA, UvrB, and UvrC proteins (9). Assay reactions were conducted according to Huang et al. (10) for the human enzyme and Sancar and Rupp (9) for the E.coli enzyme. The level of excision was quantitated by scanning the sequencing gel with an Ambis scanner. The area covering the 25-30 nt was scanned for the human excinuclease and the intensity of the $12 \mathrm{nt}$ band was taken to be the measure of the $E$.coli excinuclease activity. The final precent excision for each experiment was averaged over two independent readings from the Ambis scanner.

\section{RESULTS AND DISCUSSION}

Figure 1 (top panel) shows the three substrates used in this study. Excision of these lesions by the $E$.coli excinuclease is expected to release a radiolabeled 12 mer from the $G 1$ and $G 2$ substrates and generate a $63 \mathrm{nt}$ long labeled fragment from the G3 substrate. Figure 1 (bottom panel) shows that this is indeed the case except that with the G3 substrate a 12 mer is also seen due to the contaminating G1 substrate (see 'Materials and Methods'). Figure 1 also reveals an important contrast between the human and $E$.coli excinucleases: the human excinuclease prefers the G2 substrate which is excised rather poorly by the E.coli enzyme (lane 3 vs 7, see ref. 1). Both the E.coli and human enzymes were tested under a variety of conditions. In the case of human excinuclease, different preparations of HeLa CFE's were used in the excision assay, whereas in the case of E.coli the Uvr A, B, C proteins were stored for different lengths of time. In view of these various conditions it is therefore not surprising to see a spectrum of excision levels (Table 1). In spite of the variations of the actual excison levels between experiments, it is important to point out that the relative extents of repair of the three substrates remained nearly constant. Furthermore, it is evident from Table 1 that the human and $E$.coli enzymes have opposing substrate preferences within this sequence context.

In vitro the effect of sequence context on repair has been measured only with the E.coli excinuclease. Jones and Yeung (11) reported that the sequence context was an important determinant in what side (furan vs pyrone) was incised in psoralen-interstrand crosslinks. Thomas et al. (12) found that, in general, the excision of UV photoproducts and 4-nitroquinoline oxide-guanine adducts was sequence-independent; however it was found that a subclass of both lesions was repaired at a slower rate although no obvious consensus sequence could be inferred for the slow-repaired sites. Svoboda et al. (13) systematically varied the sequence within $10 \mathrm{bp}$ around a $\mathrm{T}<>\mathrm{T}$ and found that, essentially, the repair rate was sequence-independent although the repair rates at the two extremes differed by nearly a factor of 2. The most dramatic sequence effect on Uvr (A)BC excinulcease reported to date is that of Seeberg and Fuchs (1) on the repair of $\mathrm{G} 1, \mathrm{G} 2$, and G3 substrates used in this report. By normalizing the excision signals of the other two substrates to that of the optimal G1 substrate, the three lesions were excised at $100 \%, 15 \%$, and $58 \%$, respectively.

In contrast to the studies on E.coli excinuclease, this is the first study on the effect of sequence context on repair by the human 
excinuclease. Based on this result, we predict that the human excinuclease has different sequence preference compared with the prokaryotic enzyme even when the mutagenesis spectra of the two organisms are similar or identical.

\section{ACKNOWLEDGEMENTS}

This work was supported by the NIH grant GM32833 and by a Human Frontier Science Program Organization. D.M. is supported by a fellowship (DRG-1319) from the Cancer Research Fund of the Damon Runyon-Walter Winchell Foundation.

\section{REFERENCES}

1. Seeberg, E., and Fuchs, R. P. P. (1990) Proc. Natl Acad. Sci. USA 87, $191-194$

2. Thomas, D. C., Veaute, X., Kunkel, T. A., and Fuchs, R. P. P. (1994) Proc. Natl Acad. Sci. USA 91, 7752-7756.

3. Koffel-Schwartz, N., Verdier, J. M., Bichara, M., Freund, A. M., Daune, M. P., and Fuchs, R. P. P. (1984) J. Mol. Biol. 177, 33-51.

4. Burnouf, D., Koehl, P., and Fuchs, R. P. P. (1989) Proc. Natl Acad. Sci. USA 86, 4147-4151.

5. Bertrand-Burggraf, E., Kemper, B., and Fuchs, R. P. P. (1994) Mutation Res. 314, 287-295.

6. Koehl, P. Burnouf, D., and Fuchs, R. P. P. (1989) J. Mol. Biol. 207, 355-364.

7. Fuchs, R. (1984) J. Mol. Biol. 177, 173-180.

8. Manley, J. L., Fire, A., Cano, A., Sharp, P. A., and Gefter, M. L. (1980) Proc. Natl Acad. Sci. USA 77, 3855-3859.

9. Sancar, A. and Rupp, W. D. (1983) Cell 33, 249-260.

10. Huang, J. C., Svoboda, D. L., Reardon, J. T., and Sancar, A. (1992) Proc. Natl Acad. Sci. USA 89, 3664-3668.

11. Jones, B. K., and Yeung, A. T. (1990) J. Biol. Chem. 265, 3489-3496.

12. Thomas, D. C., Husain, I., Chaney, S. G., Panigrahi, G. B., and Walker, I. G. (1991) Nucleic Acids Res. 19, 365-370.

13. Svoboda, D. L., Smith, C. A., Taylor, J.-S., and Sancar, A. (1993) J. Biol. Chem. 268, 10694-10700. 